\title{
UN THÉORÈME DE POINT FIXE SUR LES ESPACES $L^{p}$
}

\author{
MARC BOURDON
}

\begin{abstract}
We establish a fixed point theorem for group actions on $L^{p}$-spaces, which generalizes a theorem of Żuk and of Ballmann-Świątkowski to the case $p \neq 2$.
\end{abstract}

2010 Mathematics Subject Classification: 20F65, 31C45, 47H10, 58E40.

Key words: fixed point theorem, actions of groups on Banach spaces.

\section{Introduction}

On appelle espace $L^{p}(p \in[1,+\infty))$ tout espace de Banach de la forme $L^{p}(\mathbb{R}, \nu)$, où $\nu$ est une mesure borélienne sur $\mathbb{R}$.

Soit $\Gamma$ un groupe dénombrable et soit $p \in[1,+\infty)$. On dit que $\Gamma$ possède la propriété de point fixe sur les espaces $L^{p}$ si toute action isométrique de $\Gamma$ sur un espace $L^{p}$ possède un point fixe global. Pour $p=2$ cette propriété équivaut à la propriété $(\mathrm{T})$ de Kazhdan (voir [3]). Une action isométrique de $\Gamma$ sur un espace vectoriel normé $V$ est dite propre si pour toute partie bornée $B \subset V$ le cardinal des $g \in \Gamma$ tels que $g B \cap B \neq \emptyset$ est fini.

Les réseaux des groupes de Lie simples de rang supérieur ont la propriété de point fixe sur tous les espaces $L^{p}, p \in(1,+\infty)$, [1]. A l'opposé les groupes de Coxeter, les réseaux de $\mathrm{SO}(n, 1)$, de $\mathrm{SU}(n, 1)$, les groupes moyennables, possèdent, pour tout $p \in[1,+\infty)$, une action isométrique propre sur un espace $L^{p}[\mathbf{9}],[\mathbf{8}]$. Entre ces deux situations extrêmes se situent plusieurs groupes à comportements intermédiaires. C'est le cas des groupes hyperboliques de Kazhdan (dont les réseaux cocompacts de $\operatorname{Sp}(n, 1)$ sont des exemples). En effet, les groupes Gromov hyperboliques possèdent une action isométrique propre sur un espace $L^{p}$ pour $p$ assez grand [25] (voir aussi [5]), et les groupes de Kazhdan ont la propriété de point fixe sur les espaces $L^{2}$.

Le but de ces notes est de présenter un critère qualitatif qui implique la propriété de point fixe sur les espaces $L^{p}$. Le résultat principal (Théorème 0.1 ) est un théorème de point fixe qui généralise à $p \neq 2$ 
un théorème de A. Żuk [26] et de Ballmann-Świa̧tkowski [2], et qui est implicite dans l'article $[\mathbf{1 3}]$ de M. Gromov.

\subsection{Présentation des résultats.}

Rappelons d'abord quelques définitions. Soit $X$ un 2-complexe simplicial, on désigne respectivement par $X_{0}$ et $X_{1}$ l'ensemble de ses sommets et de ses arêtes (non orientées). Pour $x \in X_{0}$ on appelle link de $x$ le graphe suivant noté $L(x)$ : ses sommets sont en bijection avec les arêtes de $X$ qui contiennent $x$, deux sommets de $L(x)$ sont reliés par une arête si les deux arêtes correspondantes de $X$ bordent un 2-simplexe. Géométriquement il est isomorphe au bord de l'union des 2-simplexes de $X$ qui contiennent $x$.

Soit $L$ un graphe fini et soit $p \in(1,+\infty)$. On désigne respectivement par $L_{0}$ et $L_{1}$ l'ensemble des sommets et des arêtes (non orientées) de $L$. Le $p$-laplacien de $L$, noté $\Delta_{p}$, est l'opérateur (non linéaire si $p \neq 2$ ) de $\mathbb{R}^{L_{0}}$ dans $\mathbb{R}^{L_{0}}$ défini par

$$
\forall u \in \mathbb{R}^{L_{0}}, \quad \forall s \in L_{0}:\left(\Delta_{p} u\right)(s)=\frac{1}{\operatorname{val}(s)} \sum_{t \sim s}\{u(t)-u(s)\}^{p-1},
$$

où val $(s)$ désigne la valence du sommet $s$ dans $L$ c'est-à-dire le nombre d'arêtes contenant $s$, où $t \sim s$ signifie que $t$ et $s$ sont les extrémités d'une même arête, et où par convention $\{x\}^{p-1}=x^{p-1}$ si $x \geq 0$, et $\{x\}^{p-1}=-|x|^{p-1}$ sinon.

On appelle valeur propre de $\Delta_{p}$ tout nombre $\lambda \in \mathbb{R}$ pour lequel il existe $u \in \mathbb{R}^{L_{0}}$ non nul avec

$$
\Delta_{p} u=\lambda\{u\}^{p-1} .
$$

On note $\lambda_{1, p}(L)$ la plus petite valeur propre non nulle de $\Delta_{p}$.

Théorème 0.1. Soit $p \in(1,+\infty)$ et soit $X$ un 2-complexe simplicial tel que pour tout $x \in X_{0}$ on ait $\lambda_{1, p}(L(x))>\frac{1}{2}$. Soit $\Gamma$ un groupe agissant sur $X$ par automorphismes simpliciaux de manière proprement discontinue et cocompacte. Alors $\Gamma$ possède la propriété de point fixe sur les espaces $L^{p}$.

Pour $p=2$ le théorème est dû à Żuk [26] lorsque l'action de $\Gamma$ sur $X$ est libre, et à Ballmann-Świątkowski [2] dans le cas général. La preuve du théorème utilise des applications harmoniques combinatoires. Cette méthode, dite de Garland, a été initiée par Garland [11], Żuk [26] et Wang [23], puis revue par Gromov [13]. Elle est encore mise en oeuvre dans $[\mathbf{1 9}],[\mathbf{2}],[\mathbf{2 4}],[\mathbf{1 0}],[\mathbf{1 5}],[\mathbf{1 4}]$. Pour un panorama du sujet on pourra consulter les articles expositoires $[\mathbf{1 2}]$, [20]. Récemment A. Naor 
et L. Silberman [18] ont établi — par des méthodes plus élaboréesque les groupes aléatoires de Gromov possèdent plusieurs propriétés de points fixes, dont celle sur les espaces $L^{p}$.

\subsection{Remarques et questions.}

1) La propriété de point fixe sur les espaces $L^{p}$ est surtout intéressante pour $p \geq 2$, en effet les propriétés suivantes sont équivalentes [1] (voir aussi $[8])$ :

- $\Gamma$ possède la propriété $(\mathrm{T})$.

- Pour tout $p \in(1,2], \Gamma$ possède la propriété de point fixe sur les espaces $L^{p}$.

- Il existe $p \in(1,2]$ tel que $\Gamma$ possède la propriété de point fixe sur les espaces $L^{p}$.

2) Dans $[\mathbf{2}$, Théorème 2], [4, $\S 1.5 .3],[\mathbf{2 2}, \S 5]$, se trouvent des exemples de groupes et de 2-complexes auxquels le Théorème 0.1 pourrait s'appliquer. Toutefois on se heurte au problème de disposer d'exemples de graphes finis qui satisfont $\lambda_{1, p}(L)>\frac{1}{2}$ pour $p$ fixé (grand). Pour cette raison le Théorème 0.1 ne permet pas, pour l'instant, de répondre à la question suivante :

Etant donné $p$ fixé (grand), existe-t'il des groupes hyperboliques qui possèdent la propriété de point fixe sur les espaces $L^{p}$ ?

Récemment A. Naor et L. Silberman [18] ont établi plusieurs théorèmes de points fixes pour les groupes aléatoires de Gromov. En particulier ils apportent une réponse positive à la question ci-dessus, en démontrant que les groupes aléatoires de Gromov possèdent cette propriété. Leur méthode - inspirée elle aussi de Gromov [13] — produit des groupes qui possèdent la propriété de point fixe sur les espaces $L^{p}$ à partir d'une famille de graphes de grandes systoles et de constantes de Poincaré $L^{p}$ uniformément bornées. N'importe quelle borne inférieure strictement positive leur suffit, contrairement au théorème de Żuk et au Théorème 0.1 qui nécessitent la borne spécifique $1 / 2$. De plus un argument simple dû à Matousek transforme une inégalité de Poincaré $L^{2}$ en une inégalité de Poincaré $L^{p}$ avec une perte sur la constante. Ceci suffit à Naor et Silberman pour obtenir leurs résultats. Cela ne permet pas d'appliquer le Théorème 0.1 car la perte est au moins égale à un facteur 4 lorsque $p>2$. Ces dernières remarques ont été proposées par l'un des rapporteurs, je l'en remercie. 
3) Soit $T$ l'arbre homogène de valence $k \geq 3$. Le bas du spectre du $p$-laplacien sur $\ell_{p}\left(T_{0}\right)$ est égal à $[6]$ :

$$
\lambda_{1, p}(T)=\frac{1}{k}\left((k-1)^{\frac{1}{p}}-1\right)^{p} .
$$

Pour $p$ fixé, il tend vers 1 lorsque $k$ tend vers $+\infty$. Cette observation suggère qu'il pourrait exister de nombreux graphes finis dont la première valeur propre non nulle du $p$-laplacien est supérieure à $\frac{1}{2}$.

4) Un graphe fini $L$ tel que pour tout $p \in(1,+\infty)$ on ait $\lambda_{1, p}(L) \geq a>0$, est nécessairement de diamêtre inférieur ou égal à 2 . En effet, en utilisant la Proposition 1.2, en élevant les deux membres de l'inégalité (1) à la puissance $1 / p$, et faisant tendre $p$ vers $+\infty$, on obtient que les éléments $u \in \mathbb{R}^{L_{0}}$ satisfont

$$
\frac{1}{2}(\sup u-\inf u) \leq \sup |d u| .
$$

En conséquence, même en dimension 2 , le Théorème 0.1 ne donne pas une nouvelle preuve du théorème de Bader-Furman-Gelander-Monod [1] qui établit la propriété de point fixe sur tous les espaces $L^{p}(p \in(1,+\infty))$ des réseaux des groupes d'automorphismes d'immeubles affines irréductibles classiques de dimension au moins 2 . Une question naturelle est de donner une preuve géométrique de ce résultat, et de le généraliser aux immeubles affines non classiques.

5) Le Théorème 0.1 est énoncé, sans démonstration et dans un cadre un peu moins général, dans mon papier [6]. La démonstration de ce cas particulier se trouve dans ma lettre [7].

\section{Organisation du papier.}

Chacun des trois premiers paragraphes du papier présente un ingrédient de la preuve du Théorème 0.1 . Le premier interprète géométriquement la première valeur propre non nulle du $p$-laplacien d'un graphe fini. Le second définit et décrit géométriquement les applications $p$-harmoniques combinatoires. Le troisième étudie l'existence d'applications $p$-harmoniques en utilisant des ultra-limites d'actions. Le théorème est démontré au quatrième paragraphe.

L'attention du lecteur est attirée sur le fait que les preuves sont plus simples et parlantes, si on suppose que le groupe $\Gamma$ agit librement sur le 2-complexe simplicial $X$ et si $X / \Gamma$ est un complexe simplicial. Nous recommandons de faire ces hypothèses lors d'une première lecture. 


\section{Remerciements.}

Je remercie H. Izeki, T. Kondo et S. Nayatani pour l'interêt qu'ils ont porté à ce travail.

\section{Inégalités de Poincaré et $p$-laplacien}

Soit $L$ un graphe fini connexe. On le suppose sans boucle c'est-à-dire que les deux extrémités de toute arête sont distinctes dans $L$. Dans ce paragraphe on interprète géométriquement la première valeur propre non nulle du $p$-laplacien de $L$, en l'identifiant à la $p$-constante de Poincaré de $L$ (Proposition 1.2). On en déduit au Corollaire 1.4 une inégalité géométrique qui est l'un des ingrédients de la preuve du Théorème 0.1.

Orientons arbitrairement chaque arête de $L$. Pour toute fonction $u: L_{0} \rightarrow \mathbb{R}$ soit $d u$ sa différentielle, c'est-à-dire la fonction de $L_{1}$ définie par $d u(a)=u\left(a_{+}\right)-u\left(a_{-}\right)$.

Définition 1.1. Soit $p \in[1,+\infty)$. La $p$-constante de Poincaré de $L$, notée $\pi_{p}(L)$, est la plus petite constante $\pi$ telle que pout toute fonction $u: L_{0} \rightarrow \mathbb{R}$ on ait

$$
\inf _{c \in \mathbb{R}} \sum_{s \in L_{0}}|u(s)-c|^{p} \operatorname{val}(s) \leq \pi \sum_{a \in L_{1}}|d u(a)|^{p} .
$$

Rappelons que $\lambda_{1, p}(L)$ désigne la plus petite valeur propre non nulle du $p$-laplacien de $L$ (voir l'introduction pour les définitions). On va établir la

Proposition 1.2. Pour $p \in(1,+\infty)$ on a $\lambda_{1, p}(L)=\frac{1}{\pi_{p}(L)}$.

Pour cela on introduit quelques objets et notations. Notons $\mu$ la mesure sur $L_{0}$ définie par $\mu(\{s\})=\operatorname{val}(s)$ pour tout $s \in L_{0}$. On désigne par $\|\cdot\|_{\mu}$ la norme suivante sur $\mathbb{R}^{L_{0}}$ :

$$
\|u\|_{\mu}^{p}=\sum_{s \in L_{0}}|u(s)|^{p} \operatorname{val}(s),
$$

et $\operatorname{par}\langle\cdot, \cdot\rangle_{\mu}$ le produit scalaire :

$$
\langle u, v\rangle_{\mu}=\sum_{s \in L_{0}} u(s) v(s) \operatorname{val}(s) .
$$

La $p$-norme de $d u$ est définie par :

$$
\|d u\|_{p}^{p}=\sum_{a \in L_{1}}\left|u\left(a_{+}\right)-u\left(a_{-}\right)\right|^{p} .
$$


Soit $\Psi: \mathbb{R}^{L_{0}} \backslash\{0\} \rightarrow \mathbb{R}$ définie par

$$
\Psi(u)=\frac{\|d u\|_{p}^{p}}{\|u\|_{\mu}^{p}} .
$$

Lemme 1.3. Les valeurs propres de $\Delta_{p}$ sont précisément les valeurs critiques de $\Psi$.

Preuve: Posons $\varphi(u)=\|d u\|_{p}^{p}$. On a pour $h \in \mathbb{R}^{L_{0}}$ et $u \in \mathbb{R}^{L_{0}} \backslash\{0\}$ :

$$
D_{u} \Psi(h)=\frac{1}{\|u\|_{\mu}^{p}} D_{u} \varphi(h)-\frac{p}{\|u\|_{\mu}^{2 p}}\left\langle h,\{u\}^{p-1}\right\rangle_{\mu}\|d u\|_{p}^{p} .
$$

De plus on a :

$$
\begin{aligned}
D_{u} \varphi(h) & =p \sum_{a \in L_{1}}\left\{u\left(a_{+}\right)-u\left(a_{-}\right)\right\}^{p-1}\left(h\left(a_{+}\right)-h\left(a_{-}\right)\right) \\
& =\frac{p}{2} \sum_{s \sim t}\{u(s)-u(t)\}^{p-1}(h(s)-h(t)) \\
& =p \sum_{s \sim t} h(s)\{u(s)-u(t)\}^{p-1} \\
& =p \sum_{s \in L_{0}} h(s) \sum_{t \sim s}\{u(s)-u(t)\}^{p-1} \\
& =p\left\langle h, \Delta_{p} u\right\rangle_{\mu} .
\end{aligned}
$$

Finalement, on obtient :

$$
D_{u} \Psi(h)=\frac{p}{\|u\|_{\mu}^{p}}\left\langle h, \Delta_{p} u-\Psi(u)\{u\}^{p-1}\right\rangle_{\mu} .
$$

Ainsi toute valeur critique de $\Psi$ est une valeur propre de $\Delta_{p}$.

Réciproquement soit $\lambda$ une valeur propre de $\Delta_{p}$ et soit $u \neq 0$ satisfaisant $\Delta_{p} u=\lambda\{u\}^{p-1}$. D'après la formule de la différentielle de $\Psi$, pour montrer que $\lambda$ est une valeur critique de $\Psi$ il suffit de voir que $\lambda=\Psi(u)$. L'égalité $\Delta_{p} u=\lambda\{u\}^{p-1}$ entraîne que

$$
\left\langle u, \Delta_{p} u\right\rangle_{\mu}=\lambda\left\langle u,\{u\}^{p-1}\right\rangle_{\mu} .
$$

Clairement le membre de droite est égal à $\lambda\|u\|_{\mu}^{p}$. Celui de gauche est égal $\|d u\|_{p}^{p}$ (par un calcul similaire à celui de $\left.D_{u} \varphi(h)\right)$. D'où l'égalité cherchée.

Preuve de la Proposition 1.2: D'après le Lemme 1.3 et sa définition, $\lambda_{1, p}(L)$ est la plus petite valeur critique non nulle de $\Psi$. 
Soit $u: L_{0} \rightarrow \mathbb{R}$ une fonction quelconque. Par stricte convexité la fonction

$$
c \in \mathbb{R} \mapsto\|u-c\|_{\mu}^{p},
$$

atteint sa borne inférieure en un unique nombre réel noté $b_{p}(u)$ et appelé le $p$-barycentre de $u$. Un calcul montre que

$$
b_{p}(u)=0 \Longleftrightarrow \sum_{s \in L_{0}}\{u(s)\}^{p-1} \operatorname{val}(s)=0 .
$$

Donc $M_{p}:=\left\{u \in \mathbb{R}^{L_{0}} ; b_{p}(u)=0\right\}$ est une sous-variété (singulière en 0 ) de $\mathbb{R}^{L_{0}}$. Remarquons que par définition

$$
\frac{1}{\pi_{p}(L)}=\inf _{u \in M_{p} \backslash\{0\}} \Psi(u) .
$$

Donc pour établir la proposition il suffit de vérifier que les valeurs critiques non nulles de $\Psi$ sont égales aux valeurs critiques de la restriction de $\Psi$ à $M_{p} \backslash\{0\}$.

Soit $u \in M_{p} \backslash\{0\}$. En ajoutant à $u$ une fonction constante on ne modifie pas sa différentielle $d u$ mais on augmente $\|u\|_{\mu}$, donc une valeur critique de la restriction de $\Psi$ à $M_{p} \backslash\{0\}$ est aussi une valeur critique de $\Psi$.

Réciproquement soit $u \in \mathbb{R}^{L_{0}} \backslash\{0\}$ un point critique de $\Psi$ avec $\Psi(u) \neq$ 0 . En considérant à nouveau la variation de $\Psi$ dans la direction des fonctions constantes, on voit que $u$ appartient à $M_{p}$.

Corollaire 1.4. Soit $p \in(1,+\infty)$ et soit $V$ un espace $L^{p}$. Pour toute application $\varphi: L_{0} \rightarrow V$ on a

$$
\inf _{v \in V} \sum_{s \in L_{0}}\|\varphi(s)-v\|_{V}^{p} \operatorname{val}(s) \leq \frac{1}{\lambda_{1, p}(L)} \sum_{a \in L_{1}}\left\|\varphi\left(a_{+}\right)-\varphi\left(a_{-}\right)\right\|_{V}^{p} .
$$

Preuve: Ecrivons $V=L^{p}(\mathbb{R}, \nu)$. Si $\nu$ est une masse de Dirac alors l'énoncé du corollaire découle trivialement de la Proposition 1.2 et de l'inégalité (1). Le cas général s'en déduit en intégrant l'inégalité (1) par rapport à $\nu$.

\section{Applications $p$-harmoniques}

Suivant M. Gromov [13, §3.11], on développe un formalisme harmonique adapté. Les techniques sont très élémentaires, elles reposent sur la notion de $p$-barycentre dont la définition est la suivante. 
Définition 2.1. Soit $p \in[1,+\infty)$, soit $V$ un espace vectoriel normé quelconque, soit $\left\{v_{i}\right\}_{1 \leq i \leq n}$ une famille d'éléments de $V$ et soit $\left\{\alpha_{i}\right\}_{1 \leq i \leq n}$ une famille de nombres strictement positifs. On appelle $p$-barycentre $d u$ système $\left\{\left(v_{i}, \alpha_{i}\right)\right\}_{1 \leq i \leq n}$ tout minimum de la fonction convexe

$$
v \in V \mapsto \sum_{i=1}^{n}\left\|v-v_{i}\right\|_{V}^{p} \alpha_{i}
$$

Observons que dans un espace vectoriel normé strictement convexe, le $p$-barycentre (s'il existe) est unique.

Soit à présent $X$ un 2-complexe simplicial, et soit $\Gamma$ un groupe agissant sur $X$ par automorphismes simpliciaux, de manière proprement discontinue et cocompacte. Pour un simplexe (non orienté) $\sigma \subset X$ on note $\Gamma_{\sigma}$ le stabilisateur de $\sigma$ dans $\Gamma$. Soit $V$ un espace vectoriel normé et soit $\Gamma \curvearrowright V$ une action isométrique de $\Gamma$ sur $V$. Considérons l'espace affine $\mathcal{E}=\left\{\varphi: X_{0} \rightarrow V ; \varphi\right.$ est $\Gamma$-équivariante $\}$. Il est naturellement isomorphe au produit

$$
\prod_{x \in \Xi_{0}} V^{\Gamma_{x}}
$$

où $\Xi_{0}$ désigne un système de représentants des orbites de $\Gamma$ dans $X_{1}$, et où $V^{\Gamma_{x}} \subset V$ est l'ensemble des vecteurs invariants par $\Gamma_{x}$. Pour $x \in X_{0}$ l'espace $V^{\Gamma_{x}}$ est non vide car $-\Gamma_{x}$ étant un groupe fini- le barycentre affine d'une orbite quelconque de $\Gamma_{x}$ dans $V$ appartient à $V^{\Gamma_{x}}$.

Soit $p \in[1,+\infty)$. Pour $\varphi \in \mathcal{E}$ on définit sa $p$-énergie, notée $E(\varphi)$, de la façon suivante. Soit $\Xi_{1} \subset X_{1}$ un système de représentants des orbites de $\Gamma$ dans $X_{1}$. On pose (comparer avec [15, p. 157]) :

$$
E(\varphi)=\sum_{e \in \Xi_{1}}\left\|\varphi\left(e_{+}\right)-\varphi\left(e_{-}\right)\right\|_{V}^{p} \frac{\operatorname{val}(e)}{\left|\Gamma_{e}\right|}
$$

où val $(e)$ est la valence de l'arête $e$ (c'est-à-dire le nombre de 2-simplexes de $X$ contenant $e$ ). Elle est indépendante du choix de $\Xi_{1}$. La proposition suivante est immédiate.

Proposition 2.2. L'action $\Gamma \curvearrowright V$ possède un point fixe global si et seulement si il existe $\varphi \in \mathcal{E}$ avec $E(\varphi)=0$.

Elle motive la

Définition 2.3. On dit que $\varphi \in \mathcal{E}$ est $p$-harmonique si $E(\varphi)=\inf _{\psi \in \mathcal{E}} E(\psi)$.

La prochaine proposition les caractérise localement : 
Proposition 2.4. Une application $\varphi \in \mathcal{E}$ est p-harmonique si et seulement si pour tout $x \in X_{0}, \varphi(x)$ est un p-barycentre du système $\{(\varphi(y)$, $\operatorname{val}([x y])\}_{y \sim x}$.

Preuve: On suppose d'abord que $X / \Gamma$ est sans boucle, c'est-à-dire que pour tout $x \in X_{0}$, toute arête de $X$ rencontre l'orbite $\Gamma \cdot x$ en au plus une extrémité. Le cas général s'y ramènera grâce à une astuce.

Supposons qu'il existe $x \in X_{0}$ tel que $\varphi(x)$ ne soit pas un $p$-barycentre du système $\left\{(\varphi(y), \operatorname{val}([x y])\}_{y \sim x}\right.$, et montrons que $\varphi$ n'est pas $p$-harmonique.

En modifiant de manière équivariante $\varphi$ le long de $\Gamma \cdot x$, seule change la partie de $E(\varphi)$ indexée par les arêtes de $\Xi_{1}$ dont l'une des extrémités appartient à $\Gamma \cdot x$. Remarquons que $E(\varphi)$ ne dépend pas du choix de $\Xi_{1}$. Soit $X_{1, x}$ l'ensemble des arêtes de $X$ contenant $x$. Puisque $X / \Gamma$ est sans boucle on peut choisir $\Xi_{1}$ de manière à ce qu'il contienne un système de représentants des orbites de $\Gamma_{x}$ dans $X_{1, x}$, noté $\Xi_{1, x}$. Alors aucune des arêtes de $\Xi_{1} \backslash \Xi_{1, x}$ ne rencontre l'orbite $\Gamma \cdot x$. Donc en modifiant de manière équivariante $\varphi$ le long de $\Gamma \cdot x$, seule change la partie de $E(\varphi)$ indexée par $\Xi_{1, x}$. Ecrivons $X_{1, x}=\bigsqcup_{e \in \Xi_{1, x}} \Gamma_{x} \cdot e$, pour toute fonction $\Gamma$-invariante $f$ de $X_{1}$ on a :

$$
\sum_{e \in X_{1, x}} f(e)=\sum_{e \in \Xi_{1, x}} f(e)\left|\Gamma_{x} \cdot e\right|=\left|\Gamma_{x}\right| \sum_{e \in \Xi_{1, x}} f(e) \frac{1}{\left|\Gamma_{e}\right|} .
$$

Prenons $f(e)=\left\|\varphi\left(e_{+}\right)-\varphi\left(e_{-}\right)\right\|_{V}^{p} \operatorname{val}(e)$, on obtient que la partie de $E(\varphi)$ indexée par $\Xi_{1, x}$ vaut :

$$
\sum_{e \in \Xi_{1, x}}\left\|\varphi\left(e_{+}\right)-\varphi\left(e_{-}\right)\right\|_{V}^{p} \frac{\operatorname{val}(e)}{\left|\Gamma_{e}\right|}=\frac{1}{\left|\Gamma_{x}\right|} \sum_{y \in X_{0}, y \sim x}\|\varphi(x)-\varphi(y)\|_{V}^{p} \operatorname{val}([x y]) .
$$

Puisque $X / \Gamma$ est sans boucle l'orbite $\Gamma \cdot x$ ne rencontre pas les voisins de $x$. Si $\varphi(x)$ n'est pas un $p$-barycentre de $\left\{(\varphi(y), \operatorname{val}([x y])\}_{y \sim x}\right.$, on peut donc modifier $\varphi$ de manière équivariante le long de $\Gamma \cdot x$ et diminuer strictement la valeur du membre de droite de (2). On décroit ainsi strictement l'énergie, donc $\varphi$ n'est pas $p$-harmonique.

Réciproquement si pour tout $x \in X_{0}, \varphi(x)$ est le $p$-barycentre du système $\left\{(\varphi(y), \operatorname{val}([x y])\}_{y \sim x}\right.$, alors en utilisant l'égalité $(2)$ on obtient que $\varphi$ est un point critique de l'énergie. Or la fonction énergie est convexe sur l'espace affine $\mathcal{E}$. Donc $E(\varphi)$ est miminale.

Dans le cas général (c'est-à-dire si on admet les boucles) on considère le revêtement d'ordre 2 de $X_{1}$, noté $\overrightarrow{X_{1}}$, constitué des arêtes orientées de $X$. Soit $\overrightarrow{\Xi_{1}}$ un système de représentants des orbites de $\Gamma$ dans $\overrightarrow{X_{1}}$. On 
voit facilement que

$$
E(\varphi)=\frac{1}{2} \sum_{\vec{e} \in \vec{\Xi}_{1}}\left\|\varphi\left(\vec{e}_{+}\right)-\varphi\left(\vec{e}_{-}\right)\right\|_{V}^{p} \frac{\operatorname{val}(e)}{\left|\Gamma_{\vec{e}}\right|},
$$

où $\Gamma_{\vec{e}}$ désigne le stabilisateur dans $\Gamma$ de l'arête orientée $\vec{e}$.

Soit $X_{0}^{-}$et $X_{0}^{+}$deux copies de $X_{0}$, et soit $Y$ le revêtement double du 1-squelette de $X$ défini comme suit : $Y_{0}=X_{0}^{-} \sqcup X_{0}^{+}, Y_{1}=\overrightarrow{X_{1}}$, et les extrémités dans $Y_{0}$ de toute arête $\vec{e} \in Y_{1}=\overrightarrow{X_{1}}$ sont $\vec{e}_{-} \in X_{0}^{-}$et $\vec{e}_{+} \in$ $X_{0}^{+}$. Le groupe $\Gamma$ agit sur $Y$ et le quotient $Y / \Gamma$ est sans boucle. Toute application $\varphi: X_{0} \rightarrow V$ se relève à $Y_{0}$ en l'application $\Gamma$-équivariante $\tilde{\varphi}$ définie par $\tilde{\varphi}\left(x_{ \pm}\right)=\varphi(x)$. D'après $(3)$ on a $E(\tilde{\varphi})=2 E(\varphi)$, en effet $\overrightarrow{\Xi_{1}}$ est un sytème de représentants des orbites de $\Gamma$ dans $Y_{1}$.

Inversement étant donnée une application $\Gamma$-équivariante $\psi: Y_{0} \rightarrow V$, on lui associe une application $\bar{\psi} \in \mathcal{E}$ par la formule

$$
\bar{\psi}(x)=\frac{1}{2}\left(\psi\left(x_{+}\right)+\psi\left(x_{-}\right)\right) .
$$

Puisque l'application $v \in V \mapsto\|v\|_{V}^{p}$ est convexe on a :

$$
E(\bar{\psi}) \leq \frac{1}{2} E(\psi)
$$

En effet, cela découle du fait que pour $x, y \in X_{0}$ avec $x \sim y$ :

$$
\begin{aligned}
\|\bar{\psi}(x)-\bar{\psi}(y)\|_{V}^{p} & =\left\|\frac{1}{2}\left(\psi\left(x_{+}\right)-\psi\left(y_{-}\right)+\psi\left(x_{-}\right)-\psi\left(y_{+}\right)\right)\right\|_{V}^{p} \\
& \leq \frac{1}{2}\left(\left\|\psi\left(x_{+}\right)-\psi\left(y_{-}\right)\right\|_{V}^{p}+\left\|\psi\left(x_{-}\right)-\psi\left(y_{+}\right)\right\|_{V}^{p}\right) .
\end{aligned}
$$

Supposons maintenant qu'il existe $x \in X_{0}$ tel que $\varphi(x)$ ne soit pas un $p$-barycentre du système $\left\{(\varphi(y), \operatorname{val}([x y])\}_{y \sim x}\right.$. Alors $\tilde{\varphi}\left(x_{+}\right)$n'est pas non plus un $p$-barycentre du système $\left\{\left(\tilde{\varphi}\left(y_{-}\right) \text {, val }([x y])\right\}_{y_{-} \sim x_{+}}\right.$. Puisque le quotient $Y / \Gamma$ est sans boucle, il existe une application $\Gamma$-équivariante $\psi: Y_{0} \rightarrow V$ telle que $E(\psi)<E(\tilde{\varphi})=2 E(\varphi)$. Alors l'application $\bar{\psi}$ appartient à $\mathcal{E}$ et satisfait $E(\bar{\psi}) \leq \frac{1}{2} E(\psi)<E(\varphi)$ d'après (4). Ainsi $\varphi$ n'est pas $p$-harmonique.

Réciproquement soit $\varphi \in \mathcal{E}$ telle que pour tout $x \in X_{0}, \varphi(x)$ soit un $p$-barycentre du système $\left\{(\varphi(y), \operatorname{val}([x y])\}_{y \sim x}\right.$. Alors l'application $\tilde{\varphi}: Y_{0} \rightarrow V$ possède la propriété analogue. Puisque $Y / \Gamma$ est sans boucle $\tilde{\varphi}$ est harmonique. Pour $\psi \in \mathcal{E}$ on obtient $E(\psi)=\frac{1}{2} E(\tilde{\psi}) \geq \frac{1}{2} E(\tilde{\varphi})=E(\varphi)$. Donc $\varphi$ est $p$-harmonique. 


\section{Ultra-limites}

M. Gromov étudie dans $[\mathbf{1 3}, \S 3.6]$ le problème de l'existence d'une application harmonique en utilisant des ultra-limites d'espaces métriques et d'actions. On présente ici un résultat (Proposition 3.1) qui découle de ses idées. Dans [14], [20] des résultats similaires sont obtenus par des méthodes voisines mais un peu différentes.

Commençons par rappeler quelques définitions, on renvoit à $[\mathbf{1 6}],[\mathbf{1 7}]$ pour plus de détails. Soit $\omega$ un ultra-filtre non principal sur $\mathbb{N}$, c'est-à-dire une mesure de probabilité finiment additive sur $\mathbb{N}$ telle que

- $\omega(A)=0$ ou 1 pour tout $A \subset \mathbb{N}$,

- $\omega(A)=0$ si $A \subset \mathbb{N}$ est un ensemble fini.

Etant donnée une suite $\left(z_{n}\right)_{n \in \mathbb{N}}$ de points d'un espace topologique $Z$, on appelle $\omega$-limite de $\left(z_{n}\right)_{n \in \mathbb{N}}$ tout $z_{\infty} \in Z$ tel que pour tout ouvert $U \subset Z$ contenant $z_{\infty}$, l'ensemble des $n \in \mathbb{N}$ tels que $z_{n} \in U$ soit de $\omega$-mesure pleine. On la dénote par $\omega$ - $\lim z_{n}$. Lorsque $Z$ est un espace métrique compact toute suite possède une et une seule $\omega$-limite.

Soit $\left(V_{n}, \star_{n}\right)_{n \in \mathbb{N}}$ une suite d'espaces vectoriels normés pointés avec $\star_{n} \in V_{n}$. On appelle ultra-limite (relativement à $\omega$ ) de la suite $\left(V_{n}, \star_{n}\right)_{n \in \mathbb{N}}$ l'espace vectoriel, noté $\omega-\lim \left(V_{n}, \star_{n}\right)$ ou simplement $V_{\omega}$, défini par

$$
V_{\omega}:=\left\{\left(v_{n}\right) \in \prod_{n \in \mathbb{N}} V_{n} ; \sup _{n}\left\|v_{n}-\star_{n}\right\|_{V_{n}}<+\infty\right\} / \sim,
$$

où $\left(v_{n}\right) \sim\left(w_{n}\right)$ si et seulement si $\omega$-lim $\left\|v_{n}-w_{n}\right\|_{V_{n}}=0$. Il est muni de la norme suivante : pour tout $v_{\omega}, w_{\omega} \in V_{\omega}$ représentés par $\left(v_{n}\right),\left(w_{n}\right)$,

$$
\left\|v_{\omega}-w_{\omega}\right\|_{V_{\omega}}:=\omega-\lim \left\|v_{n}-w_{n}\right\|_{V_{n}} .
$$

L'ultra-limite $V_{\omega}$ est toujours complet (même si les $V_{n}$ ne le sont pas), voir [17, Lemme 2.4.2]. En général $V_{\omega}$ n'est pas séparable (même si les $V_{n}$ le sont).

Proposition 3.1. Soit $X, \Gamma, p, \Gamma \curvearrowright V, \mathcal{E}$ comme au paragraphe 2. On suppose que $V$ est un espace de Banach et que $\mathcal{E}$ ne contient pas d'application p-harmonique.

(i) Si $\inf _{\varphi \in \mathcal{E}} E(\varphi)>0$, alors il existe une suite $\left(\star_{n}\right)_{n \in \mathbb{N}}$ de points de $V$ telle que l'espace $V_{\omega}:=\omega-\lim \left(V, \star_{n}\right)$ possède les propriétés suivantes : l'action $\Gamma \curvearrowright V$ se prolonge en une action isométrique $\Gamma \curvearrowright V_{\omega}$ et il existe une application $\Gamma$-équivariante $p$-harmonique de $X_{0}$ dans $V_{\omega}$ d'énergie non nulle. 
(ii) Si $\inf _{\varphi \in \mathcal{E}} E(\varphi)=0$, alors il existe des suites $\left(\star_{n}\right)_{n \in \mathbb{N}}$ et $\left(\lambda_{n}\right)_{n \in \mathbb{N}}$, avec $\star_{n} \in V$ et $\lambda_{n}>0$, telles que l'espace $V_{\omega}:=\omega-\lim \left(\lambda_{n} V, \star_{n}\right)$ possède les propriétés suivantes : les actions $\Gamma \curvearrowright \lambda_{n} V$ induisent une action isométrique $\Gamma \curvearrowright V_{\omega}$ et l'infimum des p-énergies des applications $\Gamma$-équivariantes de $X_{0}$ dans $V_{\omega}$ est non nul.

Preuve: (i) Soit $\Xi_{0}$ un système de représentants des orbites de $\Gamma$ dans $X_{0}$, soit $x_{0} \in \Xi_{0}$ une origine, et soit $\varphi_{n} \in \mathcal{E}$ telle que $E\left(\varphi_{n}\right) \leq \inf _{\varphi \in \mathcal{E}} E(\varphi)+$ $2^{-n}$. On pose $\star_{n}=\varphi_{n}\left(x_{0}\right) \in V$, et on définit $V_{\omega}=\omega$ - $\lim \left(V, \star_{n}\right)$.

Puisque $\Gamma$ agit sur $X$ de manière proprement discontinue et cocompacte, il est de type fini. Soit $S$ un système fini de générateurs de $\Gamma$. On considère la fonction de déplacement (relative à $S$ ) de l'action de $\Gamma$ sur $V$ :

$$
\forall v \in V: \delta(v)=\max _{g \in S}\|g v-v\|_{V} .
$$

La $p$-énergie de $\varphi_{n}$ étant majorée indépendamment de $n, \delta\left(\star_{n}\right)$ l'est aussi. On en déduit que l'action $\Gamma \curvearrowright V$ se prolonge en une action isométrique de $\Gamma \operatorname{sur} V_{\omega}$. De même la suite $\left(\varphi_{n}\right)_{n \in \mathbb{N}}$ donne à l' $\omega$-limite une application $\Gamma$-équivariante $\varphi_{\omega}: X_{0} \rightarrow V_{\omega}$ dont l'énergie vaut $E\left(\varphi_{\omega}\right)=\omega$ - $\lim E\left(\varphi_{n}\right)$.

Montrons que $\varphi_{\omega}$ est $p$-harmonique. Soit $\psi: X_{0} \rightarrow V_{\omega}$ une application $\Gamma$-équivariante. Pour $x \in \Xi_{0}$ choisissons une suite $\left(w_{n}\right)_{n \in \mathbb{N}}$ de points de $V$ qui représente $\psi(x)$. Puisque $\psi(x)$ est $\Gamma_{x}$-invariant, on a

$$
\forall g \in \Gamma_{x}: \omega-\lim \left\|g w_{n}-w_{n}\right\|_{V}=0 .
$$

Donc en utilisant le barycentre affine on exhibe une application $\Gamma$-équivariante $\psi_{n}: X_{0} \rightarrow V$ telle que $\psi$ soit l' $\omega$-limite de la suite $\left(\psi_{n}\right)_{n \in \mathbb{N}}$. On a donc

$$
E(\psi)=\omega-\lim E\left(\psi_{n}\right) \geq \inf _{\varphi \in \mathcal{E}} E(\varphi)=E\left(\varphi_{\omega}\right) .
$$

Ainsi $\varphi_{\omega}$ est $p$-harmonique.

(ii) On utilise le lemme suivant qui est dû à Y. Shalom ([21, Lemma 6.3]).

Lemme 3.2. Supposons que $\Gamma \curvearrowright V$ n'ait pas de point fixe global. Alors pour tout $n \in \mathbb{N}$ il existe $r_{n}>0$ et $v_{n} \in V$ tel que :

$$
\delta\left(v_{n}\right) \leq \frac{r_{n}}{n}, \text { et } \forall v \in B\left(v_{n}, r_{n}\right): \delta(v) \geq \frac{r_{n}}{2 n} .
$$

Preuve du lemme: Nous reproduisons l'argument de [21] pour le confort du lecteur. Tout d'abord $\operatorname{sinf}_{v \in V} \delta(v)>0$ il suffit de choisir $v_{n}$ tel que $\delta\left(v_{n}\right) \leq 2 \inf _{v \in V} \delta(v)$ et de poser $r_{n}=n \inf _{v \in V} \delta(v)$. 
Supposons à présent que $\inf _{v \in V} \delta(v)=0$. Soit $n \in \mathbb{N}$. Il existe $w_{1} \in V$ avec $\delta\left(w_{1}\right) \leq \frac{1}{2 n}$. Si $\delta(v) \geq \frac{1}{4 n}$ pour tout $v \in B\left(w_{1}, \frac{1}{2}\right)$, on pose $v_{n}=w_{1}$ et $r_{n}=\frac{1}{2}$.

Sinon il existe $w_{2} \in B\left(w_{1}, \frac{1}{2}\right)$ avec $\delta\left(w_{2}\right) \leq \frac{1}{4 n}$. Si $\delta(v) \geq \frac{1}{8 n}$ pour tout $v \in B\left(w_{2}, \frac{1}{4}\right)$, on pose $v_{n}=w_{2}$ et $r_{n}=\frac{1}{4}$.

Cette construction par induction s'arrête après un nombre fini d'étapes. En effet si tel n'est pas le cas, elle produit une suite $\left(w_{k}\right)_{k \in \mathbb{N}}$ de points de $V$ telle que $\left\|w_{k+1}-w_{k}\right\| \leq 2^{-k}$ et $\delta\left(w_{k}\right) \leq \frac{1}{2^{k} n}$. C'est une suite de Cauchy dans $V$ qui converge vers un point fixe de l'action de $\Gamma$, ce qui contredit l'hypothèse.

Fin de la preuve de la proposition: Avec les notations du lemme posons $\lambda_{n}=\frac{n}{r_{n}} V, \star_{n}=v_{n}$ et notons $\delta_{n}$ la fonction de déplacement de l'action de $\Gamma \operatorname{sur} \lambda_{n} V$. On a alors $\delta_{n}\left(\star_{n}\right) \leq 1$ et $\delta_{n}(v) \geq \frac{1}{2}$ pour tout $v \in$ $B\left(\star_{n}, n\right) \subset \lambda_{n} V_{n}$.

Puisque $\delta_{n}\left(\star_{n}\right) \leq 1$ les actions $\Gamma \curvearrowright \lambda_{n} V$ produisent à l' $\omega$-limite une action isométrique de $\Gamma$ sur $V_{\omega}:=\omega-\lim \left(\lambda_{n} V, \star_{n}\right)$. Comme $\delta_{n}(v) \geq \frac{1}{2}$ pour tout $v \in B\left(\star_{n}, n\right) \subset \lambda_{n} V_{n}$, la fonction de déplacement de l'action de $\Gamma$ sur $V_{\omega}$ est minorée par $\frac{1}{2}$. Par suite l'infimum des $p$-énergies des applications $\Gamma$-équivariantes de $X_{0}$ dans $V_{\omega}$ est non nul.

\section{Preuve du Théorème 0.1}

La preuve du Théorème 0.1 suit la stratégie indiquée dans $[\mathbf{1 3}, \S 3.11]$. Soit $X, \Gamma, p$ qui satisfont aux hypothèses du théorème, soit $\Gamma \curvearrowright V$ une action isométrique de $\Gamma$ sur un espace $L^{p}$, et soit $\mathcal{E}$ l'espace associé comme au paragraphe 2. Dans un premier temps on suppose que $\mathcal{E}$ contient une application $p$-harmonique et on démontre que sa $p$-énergie est nulle. La Proposition 2.2 implique alors l'existence d'un point fixe global. Dans un second temps on montre que l'hypothèse de la première étape est nécessairement satisfaite.

Première étape : On suppose que $\mathcal{E}$ contient une application $p$-harmonique $\varphi$, un "calcul magique" va entraîner que sa $p$-énergie est nulle. Il s'appuie sur les expressions suivantes (dites de localisation) valables en toute généralité (comparer avec [2, Lemme 1.3], [15, Lemme 3.2]) :

Lemme 4.1. Soit $\Xi_{0} \subset X_{0}$ un système de représentants des orbites de $\Gamma$ dans $X_{0}$. On a pour tout $\varphi \in \mathcal{E}$ :

(i) $E(\varphi)=\frac{1}{2} \sum_{x \in \Xi_{0}} \frac{1}{\left|\Gamma_{x}\right|} \sum_{y \in X_{0}, y \sim x}\|\varphi(x)-\varphi(y)\|_{V}^{p} \operatorname{val}([x y])$,

(ii) $E(\varphi)=\sum_{x \in \Xi_{0}} \frac{1}{\left|\Gamma_{x}\right|} \sum_{a \in L_{1}(x)}\left\|\varphi\left(a_{+}\right)-\varphi\left(a_{-}\right)\right\|_{V}^{p}$, 
où, dans la seconde égalité, on a identifié le link de $x$ avec le bord de l'union des 2-simplexes de $X$ qui contiennent $x$, et où on a orienté de manière arbitraire chaque arête de $L_{1}(x)$.

Supposons le lemme établi et montrons que $E(\varphi)=0$. En utilisant successivement le Lemme 4.1(i), le Corollaire 1.4 combiné à la Proposition 2.4, l'hypothèse sur les $\lambda_{1, p}$, et le Lemme 4.1(ii), il vient :

$$
\begin{aligned}
E(\varphi) & =\frac{1}{2} \sum_{x \in \Xi_{0}} \frac{1}{\left|\Gamma_{x}\right|} \sum_{y \in X_{0}, y \sim x}\|\varphi(x)-\varphi(y)\|_{V}^{p} \operatorname{val}([x y]) \\
& \leq \frac{1}{2} \sum_{x \in \Xi_{0}} \frac{1}{\left|\Gamma_{x}\right|} \frac{1}{\lambda_{1, p}(L(x))} \sum_{a \in L_{1}(x)}\left\|\varphi\left(a_{+}\right)-\varphi\left(a_{-}\right)\right\|_{V}^{p} \\
& \leq \sum_{x \in \Xi_{0}} \frac{1}{\left|\Gamma_{x}\right|} \sum_{a \in L_{1}(x)}\left\|\varphi\left(a_{+}\right)-\varphi\left(a_{-}\right)\right\|_{V}^{p} \\
& =E(\varphi) .
\end{aligned}
$$

De plus la dernière inégalité est une inégalité stricte si $E(\varphi) \neq 0$. Donc certainement $E(\varphi)=0$.

Pour compléter la première étape reste à donner la :

Preuve du Lemme 4.1: Notons d'abord que le lemme est immédiat lorsque $\Gamma$ agit librement sur $X$ et lorsque $X / \Gamma$ est un complexe simplicial (dans ce cas il est plus simple de raisonner sur le complexe simplicial $X / \Gamma)$.

Dans le cas général, pour établir l'égalité (i), on considére le revêtement d'ordre 2 de $X_{1}$, noté $\overrightarrow{X_{1}}$, constitué des arêtes orientées de $X$. Soit $\overrightarrow{\Xi_{1}}$ un système de représentants des orbites de $\Gamma$ dans $\overrightarrow{X_{1}}$. On voit facilement que

$$
E(\varphi)=\frac{1}{2} \sum_{\vec{e} \in \overrightarrow{\Xi_{1}}}\left\|\varphi\left(\vec{e}_{+}\right)-\varphi\left(\vec{e}_{-}\right)\right\|_{V}^{p} \frac{\operatorname{val}(e)}{\left|\Gamma_{\vec{e}}\right|},
$$

où $\Gamma_{\vec{e}}$ désigne le stabilisateur dans $\Gamma$ de l'arête orientée $\vec{e}$. Puisque $E(\varphi)$ ne dépend pas du choix de $\vec{\Xi}_{1}$ choisissons ce dernier comme suit. Pour $x \in X_{0}$ soit $\overrightarrow{X_{1, x}} \subset \overrightarrow{X_{1}}$ l'ensemble des arêtes orientées partant de $x$. Pour $x \in \Xi_{0}$ on choisit $\overrightarrow{\Xi_{1, x}}$ un système de représentants des orbites de $\Gamma_{x}$ dans $\overrightarrow{X_{1, x}}$, et on pose

$$
\overrightarrow{\Xi_{1}}=\bigsqcup_{x \in \Xi_{0}} \overrightarrow{\Xi_{1, x}}
$$


Comme $\overrightarrow{X_{1, x}}=\bigsqcup_{\vec{e} \in \Xi_{1, x}} \Gamma_{x} \cdot \vec{e}$, on a pour toute fonction $\Gamma$-invariante $f$ de $\overrightarrow{X_{1}}$

$$
\sum_{\vec{e} \in \overline{X_{1, x}}} f(\vec{e})=\sum_{\vec{e} \in \overline{\Xi_{1, x}}} f(\vec{e})\left|\Gamma_{x} \cdot \vec{e}\right|=\left|\Gamma_{x}\right| \sum_{\vec{e} \in \overline{\Xi_{1, x}}} f(\vec{e}) \frac{1}{\left|\Gamma_{\vec{e}}\right|} .
$$

Prenons $f(\vec{e})=\left\|\varphi\left(\vec{e}_{+}\right)-\varphi\left(\vec{e}_{-}\right)\right\|_{V}^{p} \operatorname{val}(e)$ et sommons l'égalité ci-dessus sur tout les $x \in \Xi_{0}$, on obtient la formule (i).

Pour établir (ii) convenons d'appeler simplexe pointé de $X$ tout couple $(x, a)$ où $x \in X_{0}$ et $a \in L_{1}(x) \subset X_{1}$. On note $X_{S}$ l'ensemble des simplexes pointés de $X$. Etant donnée une fonction $\Gamma$-invariante $f: X_{S} \rightarrow \mathbb{R}$ la quantité

$$
\sum_{\sigma \in \Xi_{S}} f(\sigma) \frac{1}{\left|\Gamma_{\sigma}\right|}
$$

ne dépend pas du choix du système de représentants des orbites de $\Gamma$ dans $X_{S}$, noté $\Xi_{S}$.

Pour $x \in X_{0}$ (resp. $e \in X_{1}$ ) soit $X_{S, x}$ (resp. $X_{S, e}$ ) l'ensemble des simplexes pointés de la forme $(x, a)$ (resp. $(y, e))$. Soit $\Xi_{S, x}\left(\operatorname{resp} . \Xi_{S, e}\right)$ un système de représentants des orbites de $\Gamma_{x}\left(\operatorname{resp} . \Gamma_{e}\right)$ dans $X_{S, x}$ $\left(\right.$ resp. $\left.X_{S, e}\right)$. En prenant

$$
\Xi_{S}=\bigsqcup_{x \in \Xi_{0}} \Xi_{S, x}
$$

et grâce à des arguments semblables à ceux utilisés pour montrer l'égalité (i), on obtient :

$$
\sum_{\sigma \in \Xi_{S}} f(\sigma) \frac{1}{\left|\Gamma_{\sigma}\right|}=\sum_{x \in \Xi_{0}} \frac{1}{\left|\Gamma_{x}\right|} \sum_{\sigma \in X_{S, x}} f(\sigma)
$$

De même en prenant

$$
\Xi_{S}=\bigsqcup_{e \in \Xi_{1}} \Xi_{S, e}
$$

on obtient :

$$
\sum_{\sigma \in \Xi_{S}} f(\sigma) \frac{1}{\left|\Gamma_{\sigma}\right|}=\sum_{e \in \Xi_{1}} \frac{1}{\left|\Gamma_{e}\right|} \sum_{\sigma \in X_{S, e}} f(\sigma)
$$

Pour $\sigma=(x, a)$ posons $f(\sigma)=\left\|\varphi\left(a_{+}\right)-\varphi\left(a_{-}\right)\right\|_{V}^{p}$. Les relations $(5),(6)$ entraînent l'égalité (ii). 
Seconde étape : On suppose ici qu'il n'existe pas d'application $p$-harmonique dans $\mathcal{E}$. On va aboutir à une contradiction. On distingue deux cas.

1) $\inf _{\varphi \in \mathcal{E}} E(\varphi)>0$. D'après la Proposition 3.1(i), il existe une suite $\left(\star_{n}\right)_{n \in \mathbb{N}}$ de points de $V$ telle que l'espace $V_{\omega}:=\omega-\lim \left(V, \star_{n}\right)$ possède les propriétés suivantes : l'action $\Gamma \curvearrowright V$ se prolonge en une action isométrique $\Gamma \curvearrowright V_{\omega}$ et il existe une application $\Gamma$-équivariante $p$-harmonique de $X_{0}$ dans $V_{\omega}$ d'énergie non nulle. Soit $\varphi_{\omega}$ une telle application. Par passage à l'w-limite les inégalités larges persistent, donc le Corollaire 1.4 est vrai pour $V_{\omega}$. En appliquant la première étape à $V_{\omega}$ et $\varphi_{\omega}$ on obtient $E\left(\varphi_{\omega}\right)=0$, contradiction.

2) $\inf _{\varphi \in \mathcal{E}} E(\varphi)=0$. D'après la Proposition 3.1(ii), il existe des suites $\left(\star_{n}\right)_{n \in \mathbb{N}}$ et $\left(\lambda_{n}\right)_{n \in \mathbb{N}}$ telles que l'espace $V_{\omega}:=\omega$-lim $\left(\lambda_{n} V, \star_{n}\right)$ possède les propriétés suivantes : les actions $\Gamma \curvearrowright \lambda_{n} V$ induisent une action isométrique $\Gamma \curvearrowright V_{\omega}$ et l'infimum des $p$-énergies des applications $\Gamma$-équivariantes de $X_{0}$ dans $V_{\omega}$ est non nul. A nouveau le Corollaire 1.4 est vrai pour $V_{\omega}$. On est ramené au premier cas de la seconde étape avec $V_{\omega}$ à la place de $V$. Contradiction.

\section{Références}

[1] U. Bader, A. Furman, T. Gelander et N. Monod, Property (T) and rigidity for actions on Banach spaces, Acta Math. 198(1) (2007), 57-105. DOI : 10.1007/s11511-007-0013-0.

[2] W. Ballmann et J. ŚwiątKowski, On $L^{2}$-cohomology and property $(\mathrm{T})$ for automorphism groups of polyhedral cell complexes, Geom. Funct. Anal. 7(4) (1997), 615-645. DOI : 10.1007/ s000390050022.

[3] B. Bekka, P. de la Harpe et A. Valette, "Kazhdan's property (T)", New Mathematical Monographs 11, Cambridge University Press, Cambridge, 2008. DOI : 10.1017/CB09780511542749.

[4] M. Bourdon, Sur les immeubles fuchsiens et leur type de quasi-isométrie, Ergodic Theory Dynam. Systems 20(2) (2000), 343-364. DOI : $10.1017 / \mathrm{S} 014338570000016 \mathrm{X}$.

[5] M. Bourdon, Cohomologie et actions isométriques propres sur les espaces $L_{p}$, in : "Geometry, Topology and Dynamics in Negative Curvature", Proceedings of the 2010 Bangalore Conference (to appear). 
[6] M. Bourdon, Constantes de Sobolev des arbres, Bull. Soc. Math. France 135(1) (2007), 93-103.

[7] M. Bourdon, Lettre manuscripte du 13 mars 2006 adressée à H. Izeki.

[8] I. Chatteru, C. Druţu et F. Haglund, Kazhdan and Haagerup properties from the median viewpoint, Adv. Math. 225(2) (2010), 882-921. DOI : 10.1016/j.aim.2010.03.012.

[9] P.-A. Cherix, F. Martin et A. Valette, Spaces with measured walls, the Haagerup property and property (T), Ergodic Theory Dynam. Systems 24(6) (2004), 1895-1908.

DOI : $10.1017 /$ S0143385704000185.

[10] J. Dymara et T. Januszkiewicz, New Kazhdan groups, Geom. Dedicata 80(1-3) (2000), 311-317. DOI : 10.1023/A:1005255003263.

[11] H. GARLAND, $p$-adic curvature and the cohomology of discrete subgroups of $p$-adic groups, Ann. of Math. (2) 97 (1973), 375-423. DOI : $10.2307 / 1970829$.

[12] É. GHys, Groupes aléatoires (d'après Misha Gromov,...), Astérisque 294 (2004), 173-204.

[13] M. Gromov, Random walk in random groups, Geom. Funct. Anal. 13(1) (2003), 73-146. DOI : 10.1007/s000390300002.

[14] H. Izeki, T. Kondo et S. Nayatani, Fixed-point property of random groups, Ann. Global Anal. Geom. 35(4) (2009), 363-379. DOI : $10.1007 / \mathrm{s} 10455-008-9139-3$.

[15] H. Izeki et S. NAyAtani, Combinatorial harmonic maps and discrete-group actions on Hadamard spaces, Geom. Dedicata 114 (2005), 147-188. DOI : 10.1007/s10711-004-1843-y.

[16] M. KAPOVICH, "Hyperbolic manifolds and discrete groups", Progress in Mathematics 183, Birkhäuser Boston, Inc., Boston, MA, 2001.

[17] B. Kleiner et B. Leeb, Rigidity of quasi-isometries for symmetric spaces and Euclidean buildings, Inst. Hautes Études Sci. Publ. Math. 86 (1997), 115-197 (1998).

[18] A. Naor et L. Silberman, Poincaré inequalities, embeddings, and wild groups, Compos. Math. 147(5) (2011), 1546-1572.

[19] P. Pansu, Formules de Matsushima, de Garland et propriété (T) pour des groupes agissant sur des espaces symétriques ou des immeubles, Bull. Soc. Math. France 126(1) (1998), 107-139. 
[20] P. Pansu, Superrigidité géométique et applications harmoniques, in : "Géométries à courbure négative ou nulle, groupes discrets et rigidités", Sémin. Congr. 18, Soc. Math. France, Paris, 2009, pp. $373-420$.

[21] Y. Shalom, Rigidity of commensurators and irreducible lattices, Invent. Math. 141(1) (2000), 1-54. DOI : 10.1007/ s002220000064.

[22] J. ŚwiA̧TKOwSKI, Some infinite groups generated by involutions have Kazhdan's property (T), Forum Math. 13(6) (2001), 741-755. DOI : $10.1515 /$ form. 2001.032 .

[23] M.-T. WANG, A fixed point theorem of discrete group actions on Riemannian manifolds, J. Differential Geom. 50(2) (1998), 249-267.

[24] M.-T. WANG, Generalized harmonic maps and representations of discrete groups, Comm. Anal. Geom. 8(3) (2000), 545-563.

[25] G. YU, Hyperbolic groups admit proper affine isometric actions on $l^{p}$-spaces, Geom. Funct. Anal. 15(5) (2005), 1144-1151. DOI : 10.1007/s00039-005-0533-8.

[26] A. ŻUk, La propriété (T) de Kazhdan pour les groupes agissant sur les polyèdres, C. R. Acad. Sci. Paris Sér. I Math. 323(5) (1996), 453-458.

[27] A. Żuk, Property (T) and Kazhdan constants for discrete groups, Geom. Funct. Anal. 13(3) (2003), 643-670.

DOI : $10.1007 / \mathrm{s} 00039-003-0425-8$.

Département Mathématiques

Université Lille 1

Bat. M2

59655 Villeneuve d'Ascq

France

E-mail address: bourdon@math.univ-lille1.fr

Primera versió rebuda el 12 d'agost de 2011, darrera versió rebuda el 12 de gener de 2012. 